\title{
Prognostic impact of C-reactive protein and alpha- fetoprotein in immunotherapy score in hepatocellular carcinoma patients treated with atezolizumab plus bevacizumab: A multicenter retrospective study
}

\section{Takeshi Hatanaka ( $\nabla$ hatanaka@qk9.so-net.ne.jp )}

Department of Gastroenterology, Gunma Saiseikai Maebashi Hospital https://orcid.org/0000-00033656-285X

\section{Satoru Kakizaki}

National Hospital Organization Takasaki General Medical Center

\section{Atsushi Hiraoka}

Gastroenterology Center, Ehime Prefectural Center Hospital

\section{Toshifumi Tada}

Department of Internal Medicine, Japan Red Cross Himeji Hospital

\section{Masashi Hirooka}

Department of Gastroenterology and Metaboligy, Ehime University Graduate Scholl of Medicine

\section{Kazuya Kariyama}

Department of Gastroenterology, Okayama City Hospital

\section{Joji Tani}

Department of Gastroenterology and Hepatology, Kagawa University

\section{Masanori Atsukawa}

Division of Gastroenterology and Hepatology, Department of Internal Medicine, Nippon Medical School

\section{Koichi Takaguchi}

Department of Hepatology, Kagawa Prefecture Central Hospital

\section{Ei Itobayashi}

Department of Gastroenterology, Asahi General Hospital

\section{Shinya Fukunishi}

Premeier Departmental Research of Medicine, Osaka Medical and Pharmaceutical University

Kunihiko Tsuji

Center of Gastroenterology, Teine Keijinkai Hospital

\section{Toru Ishikawa}

Saiseikai Niigata Hospital

Kazuto Tajiri 
Toyama University Hospital

\section{Hironori Ochi}

Matsuyama Red Cross Hospital

\section{Satoshi Yasuda}

Ogaki Municipal Hospital

\section{Hidenori Toyoda}

Ogaki Municipal Hospital

\section{Chikara Ogawa}

Takamatsu Red Cross Hospital

\section{Takashi Nishimura}

Hyogo College of Medicine

\section{Noritomo Shimada}

Otakanomori Hospital

\section{Kazuhito Kawata}

Hamamatsu University Scholl of Medicine

\section{Hisashi Kosaka}

Kansai Medical University

\section{Takaaki Tanaka}

Ehime Prefectural Central Hospital

\section{Hideko Ohama}

Osaka Medical and Pharmaceutical University

\section{Kazuhiro Nouso}

Okayama City Hospital

\section{Asahiro Morishita}

kagawa University

\section{Akemi Tsutsui}

Kagawa Prefectural Central Hospital

\section{Takuya Nagano}

Kagawa Prefectural Central Hospital: Kagawa Kenritsu Chuo Byoin

\section{Norio Itokawa}

Nippon Medical School: Nihon Ika Daigaku

\section{Tomomi Okubo}

Nippon Medical School: Nihon Ika Daigaku

\section{Taeang Arai}

Nippon Medical School: Nihon Ika Daigaku

\section{Michitaka Imai}

Saiseikai Niigata Hospital: Saiseikai Niigata Byoin

\section{Atsushi Naganuma}

National Hospital Organization Takasaki General Hospital 


\section{Yohei Koizumi}

Ehime University Graduate School of Medicine School of Medicine: Ehime Daigaku Daigakuin Igakukei Kenkyuka Igakubu

\section{Shinichiro Nakamura}

Himeji Red Cross Hospital: Himeji Sekijuji Byoin

\section{Kouji Joko}

Matsuyama Red Cross Hospital: Matsuyama Sekijuji Byoin

\section{Masaki Kaibori}

Kansai Medical University: Kansai Ika Daigaku

\section{Hiroko lijima}

Hyogo College of Medicine: Hyogo Ika Daigaku

\section{Yoichi Hiasa}

Ehime University Graduate School of Medicine School of Medicine: Ehime Daigaku Daigakuin Igakukei Kenkyuka Igakubu

\section{Takashi Kumada}

Gifu Kyoritsu Daigaku

\section{Research Article}

Keywords: CRAFITY score, immune checkpoint inhibitor, anti-programmed death ligand-1, vascular endothelial growth factor, inflammation, adverse events, C-reactive protein, alpha-fetoprotein, progressionfree survival, overall survival

Posted Date: February 15th, 2022

DOI: https://doi.org/10.21203/rs.3.rs-1318972/v1

License: (c) (1) This work is licensed under a Creative Commons Attribution 4.0 International License. Read Full License 


\section{Abstract}

Aim: This study aimed to investigate the utility of C-reactive protein (CRP) and alpha-fetoprotein (AFP) in immunotherapy (CRAFITY) score in hepatocellular carcinoma (HCC) patients receiving atezolizumab and bevacizumab (Atez/Bev).

Methods: This retrospective cohort study included a total of 297 patients receiving Atez/Bev from September 2020 to November 2021 at 17 different institutions and hospital groups in Japan. Patients with $A F P \geq 100 \mathrm{ng} / \mathrm{mL}$ and those with $\mathrm{CRP} \geq 1 \mathrm{mg} / \mathrm{dL}$ were assigned a CRAFITY score of 1 point.

Results: The patients were assigned CRAFITY scores of 0 points ( $n=147$ [49.5\%]), 1 point $(n=111$ [37.4\%]), and 2 points $(\mathrm{n}=39$ [13.1\%]). AFP $\geq 100 \mathrm{ng} / \mathrm{mL}$ and $\mathrm{CRP} \geq 1.0 \mathrm{mg} / \mathrm{dL}$ were significantly associated with progression-free survival (PFS) and overall survival (OS). The median PFS in the CRAFITY score 0, 1, and 2 groups was 11.8 months (95\% confidence interval [Cl] 6.4-not applicable [NA]), 6.5 months ( $95 \% \mathrm{Cl} 4.6$ 8.0), and 3.2 months ( $95 \% \mathrm{Cl} 1.9-5.0)$, respectively $(\mathrm{p}<0.001)$. The median OS in patients with CRAFITY score 0,1 and 2 was not reached, 14.3 months ( $95 \% \mathrm{Cl} 10.5-\mathrm{NA})$, and 11.6 months (95\% $\mathrm{Cl}$ 4.9-NA), respectively. The percentage of patients with grade $\geq 3$ liver injury, any grade of decreased appetite, any grade of proteinuria, any grade of fever, and any grade of fatigue was lowest in patients with a CRAFITY score of 0 , followed by patients with CRAFITY scores of 1 and 2 .

Conclusions: The CRAFITY score is simple and could be useful for predicting therapeutic outcomes and treatment-related adverse events.

\section{Introduction}

According to the Imbrave150 trial [1], combination therapy with atezolizumab plus bevacizumab (Atez/Bev), an anti-programmed death ligand 1 (PD-L1) inhibitor and monoclonal antibody targeting vascular endothelial growth factor (VEGF), demonstrated an advantage over the sorafenib in terms of the overall survival (OS) and progression-free survival (PFS). Based on the positive results, Atez/Bev have become the standard of care in first-line treatment in patients with advanced HCC under the recent guidelines [2-4]. However, the objective response rate (ORR) of immune monotherapy for HCC ranged from $17 \%$ to $20 \%$ [5-8] and only one-third of patients who received Atez/Bev treatment showed an objective response [1]. Numerous biomarkers, including the PD-L1 expression [9], and activated Wnt/ $\beta$ catenin signaling $[10,11]$, that may be used to assist in decision-making and guide treatment have been studied; however, the established biomarkers have not been fully validated [12]. A recent study [13] reported the utility of C-reactive protein (CRP) and alpha-fetoprotein (AFP) in immunotherapy (CRAFITY) score in patients treated with immunotherapy. However, more than one-half of patients included in this study were treated with immune monotherapy, and data about the efficacy and safety of Atez/Bev is limited. Accordingly, the aim of the current study is to investigate the utility of the CRAFITY score in HCC patients receiving Atez/Bev. 


\section{Methods}

\section{Patients}

A total of 325 patients with HCC received Atez/Bev from September 2020 to November 2021 at 17 different institutions and hospital groups in Japan. The inclusion criteria of this retrospective study were as follows: (a) HCC diagnosed based on typical enhancement on radiological imaging, including computed tomography and magnetic resonance imaging, or histologically proven in a biopsy specimen or a resected specimen obtained during the clinical course; (b) patients were treated with Atez/Bev; (c) The serum levels of AFP and CRP were measured at baseline. Among these 325 patients, baseline AFP data were missing for 28 patients. Therefore, the remaining 297 patients were included in the present study (Figure 1).

After receiving official approval, this study was conducted as a retrospective analysis of database records based on the Guidelines for Clinical Research issued by the Ministry of Health and Welfare of Japan. The study protocol was granted approval by the Institutional Ethics Committee of Ehime Prefectural Central Hospital (IRB No. 30-66) (UMIN000043219). All procedures were performed in accordance with the Declaration of Helsinki. Written informed consent was obtained from all patients.

\section{Atez/Bev treatment and the evaluation of AEs}

After obtaining written informed consent from each patient, all patients received intravenous Atez/Bev every 3 weeks. The Atez/Bev treatment is composed of atezolizumab $(1200 \mathrm{mg})$ and bevacizumab (15 $\mathrm{mg} / \mathrm{kg}$ body weight). The treatment was discontinued until the development of unacceptable or serious AEs or clinical tumor progression was observed. We used the Common Terminology Criteria for Adverse Events (CTCAE) version 5.0 to evaluate the AEs. We carried out dose interruption or discontinuation of each drug based on the guidelines for Atez/Bev treatment provided by the manufacturer.

\section{Evaluation of the tumor stage, liver function, and efficacy of Atez/Bev}

The tumor stage was determined by the Barcelona Clinic Liver Cancer (BCLC) staging system [4]. The liver function was evaluated by Child-Pugh classification and albumin-bilirubin (ALBI) score [14] and modified albumin-bilirubin (mALBI) grade [15]. The radiological response was assessed by the Response Evaluation Criteria In Solid Tumors version 1.1 (RECIST ver.1.1). The ORR was defined as the percentage of the sum of patients with a complete response (CR) or partial response (PR), and the disease control rate (DCR) was defined as the percentage of the sum of patients with $C R, P R$, and stable disease (SD). Progression-free survival was defined as the time from the day of starting Atez/Bev to the observation of clinical disease progression or death and OS was defined as the time from the day of starting Atez/Bev to death or the last visit. 


\section{CRAFITY score}

The CRAFITY score was determined by the values of AFP and CRP. According to a previous study [13], patients with AFP $\geq 100 \mathrm{ng} / \mathrm{mL}$ at baseline and those with $C R P \geq 1 \mathrm{mg} / \mathrm{dL}$ were assigned 1 point. For example, a patient with AFP $<100 \mathrm{ng} / \mathrm{mL}$ and CRP $<1 \mathrm{mg} / \mathrm{dL}$ was assigned to CRAFITY 0 points. A patient who had either AFP $\geq 100 \mathrm{ng} / \mathrm{mL}$ or CRP $\geq 1 \mathrm{mg} / \mathrm{dL}$ was assigned a CRAFITY score of 1 point, and a patient who had both AFP $\geq 100 \mathrm{ng} / \mathrm{mL}$ or CRP $\geq 1 \mathrm{mg} / \mathrm{dL}$ was assigned a CRAFITY score of 2 points.

\section{Statistical analyses}

All Statistical analyses were conducted using EZR Ver. 1.54 (Saitama Medical Center, Jichi Medical University, Saitama, Japan), which is a graphical user interface for R (The R Foundation for Statistical Computing, Vienna, Austria) [16]. Continuous data are presented as the median (interquartile range) and categorical data are presented as the number (percentage). The $\chi^{2}$-test, Fisher's exact, and MannWhitney $U$ test were used as appropriate. Cox proportional hazards regression models were used to evaluate the hazard ratio (HR). The number of explanatory variables involved in each model depends on the number of events. We included chronic liver disease, BCLC stage, AFP, CRP, treatment settings, age, and sex as explanatory variables in the analysis of factors associated with PFS. In the analysis of factors associated with OS, we used chronic liver disease, BCLC stage, AFP, CRP, and treatment settings as explanatory variables. Viral infection was defined as hepatitis $B$ virus (HBV) or hepatitis $C$ virus (HCV) infection. Age was dichotomized based on the median value. Because the value of CRP was strongly correlated with the ALBI score $(r=0.44, p<0.001$; Supplemental Figure 1$)$, we did not adopt the ALBI score as an explanatory variable to avoid multicollinearity. The results obtained with a cutoff value of ALBI score for $\mathrm{CRP} \geq 1 \mathrm{mg} / \mathrm{dL}$ are shown in Supplemental Figure 2 .

\section{Results}

Table 1 shows an overview of patient characteristics. The median age of all patients was 73.0 (68.0-78.0) years and 243 patients (81.8\%) were men. The PS was 0, 1, and 2 in 238 (80.1\%), 49 (16.5\%), and 10 patients (3.4\%), respectively. The etiology of chronic liver diseases was HCV, HBV, alcohol, and others in 97 (32.7\%), 49 (16.5\%), 57 (19.2\%), and 94 (31.6\%) patients, respectively. The Child-Pugh score was 5, 6, and $\geq 7$ in $183(61.6 \%), 96$ (32.3\%), and 18 patients (6.1\%), respectively. The median ALBI score was calculated to be -2.43 (-2.70 to -2.13) and the mALBI grades were 1, 2a, 2b, and 3 in 115 (38.7\%), 76 (25.6\%), $104(35.0 \%)$, and 2 patients $(0.7 \%)$, respectively. One hundred sixty-nine (56.9\%) and 128 patients $(43.1 \%)$ received Atez/Bev as a front line and later line treatment, respectively. The BCLC stage was classified as early, intermediate, advanced, and terminal in 17 (5.7\%), 121 (40.7\%), 155 (52.2\%), and 4 patients (1.3\%), respectively. There were 122 patients (41.1\%) with a serum AFP $\geq 100 \mathrm{ng} / \mathrm{mL}$ and 67 patients $(22.6 \%)$ with serum CRP $\geq 1.0 \mathrm{mg} / \mathrm{dL}$. Accordingly, the patients were assigned CRAFITY scores of $0(n=147[49.5 \%]), 1(n=111[37.4 \%])$, and 2 points $(n=39$ [13.1\%]). The patients with a CRAFITY score 
of 0 points showed significantly better PS, better preservation of the liver function, a higher percentage of first-line treatment, and an earlier HCC stage in comparison to those with CRAFITY scores of 1 or 2 points.

The numbers of patients with a confirmed radiological response, as assessed by RECIST ver.1.1, are shown in Table 2. Among the patients with CRAFITY scores of 0,1 , and 2 points, 119, 101, and 37 patients, respectively, showed a confirmed radiological response. The radiological response rate $(p=0.20)$ and ORR ( $p=0.80)$ were not significantly different, while a significant difference in the DCR was observed among the three groups $(p=0.029)$.

The Kaplan-Meier curves showed that the median PFS was 6.8 months (95\% Cl 6.0-8.0), with 144 events (48.8\%) detected at the time of the analysis (Figure 2a). While the median OS was not reached, the 6month, and 12-month OS rates were 89.9\% (95\% Cl 85.3-93.1) and 66.1\% (95\% Cl 55.6-74.6\%), respectively, with 52 events (17.5\%) found at the time of the analysis (Figure $2 b$ ). The results obtained from the multivariate analysis are presented in Table 3. The following factors showed a significant association with PFS: AFP $\geq 100 \mathrm{ng} / \mathrm{mL}$ (HR 1.97, 95\% Cl 1.40-2.77, $\mathrm{p}<0.001)$ and $\mathrm{CRP} \geq 1.0 \mathrm{mg} / \mathrm{dL}$ (HR $1.51,95 \% \mathrm{Cl} 1.05-2.19, \mathrm{p}=0.028)$. A statistical analysis of factors related to OS also revealed that $A F P \geq 100 \mathrm{ng} / \mathrm{mL}$ (HR 2.74, 95\% Cl 1.52-4.92, $\mathrm{p}<0.001$ ) and $\mathrm{CRP} \geq 1.0 \mathrm{mg} / \mathrm{dL}$ (HR 1.87, 95\% Cl 1.06-3.31, $\mathrm{p}=0.032$ ) were predictors of OS. A multivariate analysis was performed using the CRAFITY score as an explanatory variable, and the HRs and $95 \%$ Cls of each CRAFITY score are described in Supplemental Table 1.

The PFS and OS for each CRAFITY score are shown in Figure 3. In the CRAFITY 0, 1, and 2 points groups, the median PFS was 11.8 months (95\% Cl 6.4-not applicable [NA]), 6.5 months (95\% Cl 4.6-8.0), and 3.2 months $(95 \% \mathrm{Cl} 1.9-5.0)$, respectively, $(\mathrm{p}<0.001)$. The results of the analysis of PFS in patients with BCLC early and intermediate stage according to the CRAFITY score are shown in Supplemental Figure 2a and those of patients with BCLC advanced and terminal stage are shown in Supplemental Figure $2 \mathrm{~b}$. The median OS in patients with CRAFITY score 0 points was not reached while it was 14.3 months $(95 \% \mathrm{Cl}$ 10.5-NA) and 11.6 months ( $95 \% \mathrm{Cl}$ 4.9-NA) in patients with CRAFITY scores of 1 point and 2 points, respectively. There was a significant difference among the three groups $(p<0.001)$. The 6 -month and $12-$ month OS rates were $94.7 \%$ (95\% $\mathrm{Cl} 88.4-97.6)$ and $81.1 \%$ (95\% $\mathrm{Cl} 66.1-89.9 \%)$, respectively, in patients with CRAFITY score 0, 92.9\% (95\% Cl 85.6-96.6\%) and 63.5\% (48.3-75.3\%) in patients with CRAFITY score 1, and $63.6 \%$ (95\% $\mathrm{Cl} 44.5-77.7 \%)$ and $33.2 \%(95 \% \mathrm{Cl} 10.5-58.3 \%)$ in patients with CRAFITY score 2 . The survival curves for patients with BCLC early and intermediate stage, stratified by the CRAFITY score, are shown in Supplemental Figure 3a, while those with BCLC advanced and terminal stage are also shown in Supplemental Figure 3b.

A summary of AEs according to the CRAFITY score is shown in Table 4. The most common AEs in all patients was fatigue $(n=75,25.3 \%)$, followed by proteinuria $(n=71,23.9 \%)$, decreased appetite $(n=70$, $23.6 \%)$, hypertension $(n=58,19.5 \%)$, and liver injury $(n=40,13.5 \%)$. Significant differences were observed in grade $\geq 3$ liver injury $(p=0.036)$, any grade of decreased appetite $(p=0.002)$, any grade of proteinuria 
$(p=0.039)$, any grade of fever $(p=0.011)$, and any grade of fatigue $(p=0.032)$. The rates of these AEs were lowest in patients with a CRAFITY score of 0 , followed by patients with CRAFITY scores of 1 and 2 .

\section{Discussion}

The major finding of the present study is that AFP $\geq 100 \mathrm{ng} / \mathrm{mL}$ and CRP $\geq 1.0 \mathrm{mg} / \mathrm{dL}$ were found to be predictive factors associated with PFS and OS in patients treated with Atez/Bev. The CRAFITY score, which is composed of AFP and CRP, could stratify the OS of patients treated with Atz/Bev. Because the previous report [13] did not investigate the correlation of the CRAFITY score with PFS and AEs, we revealed that the CRAFITY score could also predict PFS and treatment-related AEs. Accordingly, the CRAFITY score was simple and useful for predicting therapeutic outcomes and treatment-related AEs. To our knowledge, this is the first report assessing the utility of the CRAFITY score in HCC patients treated with Atez/Bev.

AFP is a well-known, novel tumor biomarker, that is widely used in the clinical setting. An elevated serum level of AFP was associated with a poor prognosis across all stages of HCC [12]. Some studies revealed that AFP was a prognostic factor in patients treated with surgical resection [17], liver transplantation [18], radiofrequency ablation [19, 20], and transarterial chemoembolization [21]. In addition, AFP was also associated with a high rate of recurrence after liver transplantation [22]. Due to the prognostic significance of AFP in advanced HCC patients, the pretreatment AFP concentration has been adopted as a stratification factor in recent phase 3 trials $[1,23,24]$. With regard to the molecular HCC classes, Hoshida's HCC subclasses (S1-S3) are associated with various parameters, such as tumor size, tumor differentiation, and AFP [25]. Among these subclasses, the S2 subclass is associated with high serum AFP [25]. Moreover, the expression of AFP and EpCAM (a hepatic stem cell expression marker) were used to characterize the progenitor cell group (S2 subclass) [26]. Recently, AFP was also shown to be associated with the activation of the tumor VEGF pathway [27, 28]. VEGF reduces the therapeutic effect of immune checkpoint inhibitors (ICls) via some mechanisms, including inhibition of the maturation of dendritic cells [29, 30], stimulation of lymphocyte rolling [31, 32], intra-tumoral T-cell infiltration [33], and expansion of immunosuppressive myeloid-derived suppressor cells (MDSCs) [34, 35]. Given these previous studies, patients with high AFP levels ( $\geq 100 \mathrm{ng} / \mathrm{dL}$ ) showed a poor prognosis and shorter PFS in comparison to those with low AFP levels in the present study.

Inflammation is considered a hallmark of cancer progression and a key component of the tumor microenvironment [36,37]. C-reactive protein is an acute protein and is mainly regulated by interleukin- 6 [38]. To date, many studies reported that CRP is a novel prognostic marker in HCC patients [39-41]. Regarding ICl treatment, an elevated CRP level has been reported as an unfavorable factor in some types of cancers, including non-small cell lung cancer $[42,43]$ and melanoma $[43,44]$; however, few reports have investigated the relationship between the single determination of CRP and ICl efficacy in HCC patients. An association between CRP and immunosuppression was recently reported. CRP binds to Tcells and has a profound suppressive effect on immunity in patients $[45,46]$. CRP also regulates the 
development and suppressive actions of MDSC [47]. These previous reports support the present findings that CRP is a predictive factor for PFS and OS.

With regard to AEs, the clear mechanism underlying the association between AEs and the CRAFITY score remains unknown. One possible reason is that cancer-related symptoms, such as appetite loss, weight loss, and fatigue, are associated with not only the tumor burden or aggressive types of tumors, but also the presence of inflammation [48]. Obviously, elevated AFP was frequently observed in patients with advanced or aggressive HCC, and elevated CRP reflects the presence of inflammation. Indeed, elevated CRP is associated with AEs in melanoma patients treated with ICI [49]. Accordingly, the CRAFITY score, which is composed of AFP and CRP, could predict AEs in patients receiving Atez/Bev.

Regarding the analysis of the radiological response, although statistical significance was not observed in the analysis of the ORR, it was observed in the analysis of the DCR. A previous study [13] by Scheiner et al. showed that CRAFITY score predicted the ORR and DCR. This difference may be associated with the short observation period of the present study. A further study with long-term observation is needed to confirm the correlation between the CRAFITY score and the ORR and DCR of patients treated with Atez/Bev.

The present study was associated with some limitations. This study was conducted in a retrospective manner. Although the present study included a larger number of patients in comparison to previous studies [13], the observation period of the present study was short. Further prospective studies with longterm follow-up are warranted.

In conclusion, the CRAFITY score is simple to determine and could be useful for predicting therapeutic outcomes and treatment-related AEs.

\section{Declarations}

\section{Author contributions}

TH, SK, AH, TTad, AN, and TK conceived the study, and participated in its design and coordination. TH, SK, AH, TTad, MH, KKar, JT, MA, KTak, El, SF, KTs, TI, KTaj, HOc, SY, HT, CO, TNi, NS, KKaw, TTan, HOh, KN, $A M, A T, T N a, N I, T O, T A, M I, A N, Y K, S N, K J, H I$, and $Y H$ performed data curation. TH performed statistical analyses and interpretation. TH, SK, AH, TTad, AN, TK, HK, and MK drafted the text. All authors have read and approved the final version of the manuscript.

\section{Funding}

No financial support.

\section{Compliance with ethical standards}




\section{Conflict of interest}

Takeshi Hatanaka received lecture fees from Eisai. Atsushi Hiraoka received lecture fees from Bayer, Eisai, Eli Lilly, Otsuka, and Chugai. Takashi Kumada received lecture fees from Eisai.

\section{References}

1. Finn RS, Qin S, Ikeda M, et al. Atezolizumab plus bevacizumab in unresectable hepatocellular carcinoma. N Engl J Med. 2020;382:1894-905.

2. Gordan JD, Kennedy EB, Abou-Alfa GK, et al. Systemic therapy for advanced hepatocellular carcinoma: ASCO guideline. J Clin Oncol. 2020;38:4317-45.

3. Vogel A, Martinelli E. Updated treatment recommendations for hepatocellular carcinoma (HCC) from the ESMO Clinical Practice Guidelines. Ann Oncol. 2021;32:801-5.

4. Llovet JM, Villanueva A, Marrero JA, et al. Trial Design and Endpoints in Hepatocellular Carcinoma: AASLD Consensus Conference. Hepatology. 2021;73 Suppl 1:158-91.

5. El-Khoueiry AB, Sangro B, Yau T, et al. Nivolumab in patients with advanced hepatocellular carcinoma (CheckMate 040): an open-label, non-comparative, phase 1/2 dose escalation and expansion trial. Lancet. 2017;389:2492-502.

6. Zhu AX, Finn RS, Edeline J, et al. Pembrolizumab in patients with advanced hepatocellular carcinoma previously treated with sorafenib (KEYNOTE-224): a non-randomised, open-label phase 2 trial. Lancet Oncol. 2018;19:940-52.

7. Finn RS, Ryoo BY, Merle P, et al. Pembrolizumab as second-line therapy in patients with advanced hepatocellular carcinoma in KEYNOTE-240: A randomized, double-blind, phase III Trial. J Clin Oncol. 2020;38:193-202.

8. Lee MS, Ryoo BY, Hsu CH, et al. Atezolizumab with or without bevacizumab in unresectable hepatocellular carcinoma (G030140): an open-label, multicentre, phase 1b study. Lancet Oncol. 2020;21:808-20.

9. Li XS, Li JW, Li H, et al. Prognostic value of programmed cell death ligand 1 (PD-L1) for hepatocellular carcinoma: a meta-analysis. Biosci Rep. 2020;40.

10. Ruiz de Galarreta $M$, Bresnahan $E$, Molina-Sánchez $P$, et al. $\beta$-catenin activation promotes immune escape and resistance to anti-PD-1 therapy in hepatocellular carcinoma. Cancer Discov. 2019;9:112441.

11. Sia D, Jiao Y, Martinez-Quetglas I, et al. Identification of an immune-specific class of hepatocellular carcinoma, based on molecular features. Gastroenterology. 2017;153:812-26.

12. Llovet JM, Kelley RK, Villanueva A, et al. Hepatocellular carcinoma. Nat Rev Dis Primers. 2021;7:6.

13. Scheiner B, Pomej K, Kirstein MM, et al. Prognosis of patients with hepatocellular carcinoma treated with immunotherapy - development and validation of the CRAFITY score. J Hepatol. 2021; doi:10.1016/j.jhep.2021.09.035. 
14. Johnson PJ, Berhane S, Kagebayashi C, et al. Assessment of liver function in patients with hepatocellular carcinoma: a new evidence-based approach-the ALBI grade. J Clin Oncol. 2015;33:550-8.

15. Hiraoka A, Michitaka K, Kumada T, et al. Validation and Potential of Albumin-Bilirubin Grade and Prognostication in a Nationwide Survey of 46,681 Hepatocellular Carcinoma Patients in Japan: The Need for a More Detailed Evaluation of Hepatic Function. Liver Cancer. 2017;6:325-36.

16. Kanda Y. Investigation of the freely available easy-to-use software 'EZR' for medical statistics. Bone Marrow Transplant. 2013;48:452-8.

17. Ridder DA, Weinmann A, Schindeldecker M, et al. Comprehensive clinicopathologic study of alpha fetoprotein-expression in a large cohort of patients with hepatocellular carcinoma. Int J Cancer. 2021; doi:10.1002/ijc.33898.

18. Mazzaferro V, Sposito C, Zhou J, et al. Metroticket 2.0 Model for Analysis of Competing Risks of Death After Liver Transplantation for Hepatocellular Carcinoma. Gastroenterology. 2018;154:128-39.

19. Lee DH, Lee JM, Lee JY, et al. Radiofrequency ablation of hepatocellular carcinoma as first-line treatment: long-term results and prognostic factors in 162 patients with cirrhosis. Radiology. 2014;270:900-9.

20. N'Kontchou G, Mahamoudi A, Aout M, et al. Radiofrequency ablation of hepatocellular carcinoma: long-term results and prognostic factors in 235 Western patients with cirrhosis. Hepatology. 2009;50:1475-83.

21. Takayasu K, Arii S, Kudo M, et al. Superselective transarterial chemoembolization for hepatocellular carcinoma. Validation of treatment algorithm proposed by Japanese guidelines. J Hepatol. 2012;56:886-92.

22. Mehta N, Dodge JL, Roberts JP, et al. Validation of the prognostic power of the RETREAT score for hepatocellular carcinoma recurrence using the UNOS database. Am J Transplant. 2018;18:1206-13.

23. Kudo M, Finn RS, Qin S, et al. Lenvatinib versus sorafenib in first-line treatment of patients with unresectable hepatocellular carcinoma: a randomised phase 3 non-inferiority trial. Lancet. 2018;391:1163-73.

24. Bruix J, Qin S, Merle P, et al. Regorafenib for patients with hepatocellular carcinoma who progressed on sorafenib treatment (RESORCE): a randomised, double-blind, placebo-controlled, phase 3 trial. Lancet. 2017;389:56-66.

25. Hoshida Y, Nijman SM, Kobayashi M, et al. Integrative transcriptome analysis reveals common molecular subclasses of human hepatocellular carcinoma. Cancer Res. 2009;69:7385-92.

26. Yamashita T, Forgues M, Wang W, et al. EpCAM and alpha-fetoprotein expression defines novel prognostic subtypes of hepatocellular carcinoma. Cancer Res. 2008;68:1451-61.

27. Galle PR, Foerster F, Kudo M, et al. Biology and significance of alpha-fetoprotein in hepatocellular carcinoma. Liver Int. 2019;39:2214-29.

28. Montal R, Andreu-Oller C, Bassaganyas L, et al. Molecular portrait of high alpha-fetoprotein in hepatocellular carcinoma: implications for biomarker-driven clinical trials. Br J Cancer. 2019;121:340- 
3.

29. Oyama T, Ran S, Ishida T, et al. Vascular endothelial growth factor affects dendritic cell maturation through the inhibition of nuclear factor-kappa B activation in hemopoietic progenitor cells. $J$ Immunol. 1998;160:1224-32.

30. Gabrilovich DI, Chen HL, Girgis KR, et al. Production of vascular endothelial growth factor by human tumors inhibits the functional maturation of dendritic cells. Nat Med. 1996;2:1096-103.

31. Borgström P, Hughes GK, Hansell P, et al. Leukocyte adhesion in angiogenic blood vessels. Role of Eselectin, P-selectin, and beta2 integrin in lymphotoxin-mediated leukocyte recruitment in tumor microvessels. J Clin Invest. 1997;99:2246-53.

32. Hegde PS, Wallin JJ, Mancao C. Predictive markers of anti-VEGF and emerging role of angiogenesis inhibitors as immunotherapeutics. Semin Cancer Biol. 2018;52:117-24.

33. Wallin JJ, Bendell JC, Funke R, et al. Atezolizumab in combination with bevacizumab enhances antigen-specific T-cell migration in metastatic renal cell carcinoma. Nat Commun. 2016;7:12624.

34. Voron T, Colussi O, Marcheteau E, et al. VEGF-A modulates expression of inhibitory checkpoints on CD8+ T cells in tumors. J Exp Med. 2015;212:139-48.

35. Gabrilovich DI, Nagaraj S. Myeloid-derived suppressor cells as regulators of the immune system. Nat Rev Immunol. 2009;9:162-74.

36. Diakos $\mathrm{Cl}$, Charles KA, McMillan DC, et al. Cancer-related inflammation and treatment effectiveness. Lancet Oncol. 2014;15:e493-503.

37. Mantovani A, Allavena P, Sica A, et al. Cancer-related inflammation. Nature. 2008;454:436-44.

38. Morris-Stiff G, Gomez D, Prasad KR. C-reactive protein in liver cancer surgery. Eur J Surg Oncol. 2008;34:727-9.

39. Sieghart W, Pinter M, Hucke F, et al. Single determination of C-reactive protein at the time of diagnosis predicts long-term outcome of patients with hepatocellular carcinoma. Hepatology. 2013;57:2224-34.

40. Meischl T, Rasoul-Rockenschaub S, Györi G, et al. C-reactive protein is an independent predictor for hepatocellular carcinoma recurrence after liver transplantation. PLoS One. 2019;14:e0216677.

41. Hayashi T, Shibata M, Oe S, et al. C-reactive protein can predict dose intensity, time to treatment failure and overall survival in HCC treated with lenvatinib. PLoS One. 2020;15:e0244370.

42. Riedl JM, Barth DA, Brueckl WM, et al. C-Reactive Protein (CRP) levels in immune checkpoint inhibitor response and progression in advanced non-small cell lung cancer: A bi-center study. Cancers (Basel). 2020;12.

43. livanainen S, Ahvonen J, Knuuttila A, et al. Elevated CRP levels indicate poor progression-free and overall survival on cancer patients treated with PD-1 inhibitors. ESMO Open. 2019;4:e000531.

44. Laino AS, Woods D, Vassallo M, et al. Serum interleukin-6 and C-reactive protein are associated with survival in melanoma patients receiving immune checkpoint inhibition. $\mathrm{J}$ Immunother Cancer. $2020 ; 8$. 
45. Yoshida T, Ichikawa J, Giuroiu I, et al. C reactive protein impairs adaptive immunity in immune cells of patients with melanoma. J Immunother Cancer. 2020;8.

46. Zhang L, Liu SH, Wright TT, et al. C-reactive protein directly suppresses Th1 cell differentiation and alleviates experimental autoimmune encephalomyelitis. J Immunol. 2015;194:5243-52.

47. Jimenez RV, Kuznetsova V, Connelly AN, et al. C-Reactive Protein Promotes the Expansion of Myeloid Derived Cells With Suppressor Functions. Front Immunol. 2019;10:2183.

48. Roxburgh CS, McMillan DC. Cancer and systemic inflammation: treat the tumour and treat the host. Br J Cancer. 2014;110:1409-12.

49. Abolhassani AR, Schuler G, Kirchberger MC, et al. C-reactive protein as an early marker of immunerelated adverse events. J Cancer Res Clin Oncol. 2019;145:2625-31.

\section{Tables}

Tables 1-4 are available in the Supplemental Files section.

\section{Figures}

\section{Figure 1}

Flow chart of the selection of HCC patients treated with Atez/Bev. Atez/Bev, atezolizumab plus bevacizumab; CRP, C-reactive protein; HCC, hepatocellular carcinoma.

\section{Figure 2}

Kaplan-Meier curves for progression-free survival (a) and overall survival (b) in all patients.

\section{Figure 3}

Kaplan-Meier curves for progression-free survival (a) and overall survival (b) according to the CRAFITY score.

CRAFITY score, C-reactive protein and alpha-fetoprotein in immunotherapy.

\section{Supplementary Files}

This is a list of supplementary files associated with this preprint. Click to download. 
- AtezBevCRAFITYSupplementalTable2021Dec28.xIsx

- SuppFig1.png

- SuppFig2.png

- SuppFig3.png

- AtezBevCRAFITYTable2021Dec28.xIsx 